\title{
Automated Telephone Calls in the Follow-Up of Self-Care in Outpatients with Type 2 Diabetes: A Feasibility Study
}

\author{
Esther C. Gallegos-Cabriales ${ }^{1}$, Juana Mercedes Gutiérrez-Valverde ${ }^{1}$, \\ Bertha Cecilia Salazar-González ${ }^{1}$, Antonia M. Villarruel2, \\ Rosa Alicia Veloz-Garza1, Nicolle Marinec ${ }^{3}$,John D. Piette ${ }^{3,4}$ \\ ${ }^{1}$ School of Nursing, Universidad Autónoma de Nuevo León, Monterrey, México \\ ${ }^{2}$ School of Nursing, University of Pennsylvania, Philadelphia, PA, USA \\ ${ }^{3}$ Michigan Center for Diabetes Translational Research, University of Michigan, Ann Arbor, Michigan, USA \\ ${ }^{4}$ Center for Managing Chronic Disease, University of Michigan, Ann Arbor, Michigan, USA \\ Email: juana.gutierrezv@uanl.mx
}

How to cite this paper: Gallegos-Cabriales, E.C., Gutiérrez-Valverde, J.M., Salazar-González, B.C., Villarruel, A.M., Veloz-Garza, R.A., Marinec, N. and Piette, J.D. (2017) Automated Telephone Calls in the Follow-Up of Self-Care in Outpatients with Type 2 Diabetes: A Feasibility Study. Health, 9, 1529-1541.

https://doi.org/10.4236/health.2017.911113

Received: September 1, 2017

Accepted: October 27, 2017

Published: October 30, 2017

Copyright $\odot 2017$ by authors and Scientific Research Publishing Inc. This work is licensed under the Creative Commons Attribution International License (CC BY 4.0).

http://creativecommons.org/licenses/by/4.0/ Open Access

\begin{abstract}
Aims and objectives: To test an automated telephone service as a follow-up and support strategy for self-care in a population of adult Mexican patients with type 2 diabetes. Methods: The design was a two-group comparative design, with pre and post-intervention measurements. Patients were assigned into intervention $(n=31)$, or comparison $(n=33)$ groups. Over 12 weeks, each participant in the intervention group received automated phone calls with instructions to facilitate accomplishment of the treatment, and self-care activities. Two focus groups helped to appreciate patients' follow-up experience. Randomized grouping was performed by selecting numbers from an urn; the final sample comprised 31 (48\%) patients in the intervention group, and $33(52 \%)$ in the comparison group; these procedures were performed by the case nurse management. The study was approved by the Ethics Committee of the Autonomous University of Nuevo Leon and the University of Michigan; all of the participants signed informed consent. Results: Of the 372 programmed phone calls made to the participants, 234 were completed, representing a $62.9 \%$ response rate. Phone calls may be associated to a $0.82 \%$ decrease in the HbA1c values and to lower depression scores. HbA1c measurements decreased $0.82 \%(M=7.41-6.69 ; t=25 ; 4.11 ; p=-0.001)$ from the baseline values over the 12 -week study in the intervention group and $0.49 \%$ $(M=7.24-6.75 ; t=26 ; 2.11 ; p=0.044)$ in the comparison group. Variables related to medication intake did not exhibit differences between the baseline and second measurements, except with respect to the scale of depression.
\end{abstract}




\section{Keywords}

Diabetes, Self-Care, Technology, Information Technology, Intervention, Chronic Illness, Counseling, Self-Management, Telenursing

\section{Introduction}

Diabetes is a serious public health problem in Mexico, and the prevalence of diabetes has increased from $6.7 \%$ in 1993 to $14.42 \%$ in 2006 [1]. Approximately $95 \%$ of patients exhibit poor or very poor glycemic control due to inconsistent routine treatment [2]. Therefore, diabetes has become the most common cause of death and one of the major factors contributing to decreased quality of life in Mexico. Diabetes is also the leading cause of blindness, terminal chronic renal failure, and non-traumatic amputations. The poor overall control of diabetes and its numerous complications contribute to this disease being responsible for the greatest expenditure of the health system in Mexico [3].

\section{Background}

Ninety-five percent of patients with diabetes have type 2 diabetes (T2D). During its course, patients suffer asymptomatic mild hyperglycemic events that can lead to the discontinuation of treatment, specifically, the modifications of diet and exercise lifestyle, which are basic elements in treatment [4] [5]. Clinical research has proven the effectiveness of psycho-educational interventions in lifestyle modification and prescription treatment observation [6] [7] [8]. However, psycho-educational interventions have only a short-term effect, and new effective mid- and long-term treatment management and metabolic control strategies are imperative for the management of T2D patients.

Care provided by health professionals through telephone calls has shown to be effective in improving the self-management of chronic diseases such as T2D, representing a useful mid- and long-term follow-up mechanism [9] [10] [11] [12]. However, the health service and specialized facility infrastructure requirements of patients are not clear, and it remains unclear whether this strategy is feasible for patients with little formal education, and with limited economical resources [13] [14].

The use of mobile telephone devices has become increasingly common worldwide, and researchers are exploring how to use mobile devices as a tool to improve diabetic patient support in their environment. Currently, approximately three quarters of mobile users live in developing countries, such as Mexico, and more than $90 \%$ of the world has satellite coverage [15]. Several studies have demonstrated that communicating with patients through short message service (SMS) may improve the self-management of glycemic levels [16] [17] [18] [19]. Another innovating strategy is to take advantage of mobile telephones for automated calls. Through automated calls, patients may receive more extensive in- 
formation than through an SMS, and the information can be more individualized because the patients report selective health data during the phone call. Furthermore, automated telephone calls may be more accessible for diabetic patients with low literacy, vision impairment, or tactile sensitivity. Rigorous research on the Latin American population of the USA has reported that interventions that include phone calls improve health behavior and result in diabetic control [20] [21]. Recent studies demonstrate that diabetic and hypertensive patients in Latin America may benefit from these strategies, improving the physiological control of these diseases [22] [23].

In the state of Nuevo Leon, Mexico, outpatient routine treatment of T2D patients in health units consists of regular medical appointments. However, as in other countries, a high percentage of these patients do not have access to these consultations, which leads to a lack of self-management information and difficulty in preventing the complications of the disease. The aim of the present study was to determine the feasibility of an automated telephone call follow-up strategy for self-care in T2D adult patients from low socio-economical areas of Monterrey, Mexico. This calling system was aimed at improving adherence to treatment and glycemic control. As a pilot study, we intend to answer the following research questions:

1) How feasible is the use of an "Automated Telephone Diabetes Management" system in low-income T2D patients of the metropolitan area of Monterrey, Nuevo Leon, Mexico? Will the patients respond regularly? Are they satisfied with the service?

2) What is the effect of a physiological control and self-care behavioral system?

\section{Materials and Methods}

\subsection{Design}

The feasibility study is a randomized controlled trial with intervention and comparison groups, and measurements pre post intervention.

\subsection{Sampling and Recruitment}

Participants were recruited from three university clinics that serve a low socioeconomic population of the metropolitan area of Monterrey. Routine appointments of T2D adults in these clinics consist of a monthly medical consultation during which capillary glucose concentrations, weight, and arterial pressure are measured. Sporadically, educational meetings (support groups) are held during which food preparation and exercise routine demonstrations, as well as lower extremities examinations, are performed.

Patients who attended the clinics for medical control, as well as those asking for medical consultation during the study were invited to be part of it. One hundred and twenty three potential participants were contacted in total, and invited to join the study. Patients 18 yrs. and older with a fixed line or mobile telephone, 
were included in the intervention. Patients who were hard of hearing (i.e., those who could not follow a telephone conversation) or suffering from renal failure (verified through the clinical history) were excluded $(n=7)$.

The 123 contacted patients were interviewed finding 116 eligible subjects. Of these, only $55 \%$ were able to participate $(n=64)$. Subjects that declined to be part of the study argued having difficulties receiving phone calls due to their work, or excessive household responsibilities. Randomized grouping was performed by selecting numbers from an urn; the final sample comprised 31 (48\%) patients in the intervention group, and 33 (52\%) in the comparison group; these procedures were performed by the case nurse management. However, due to time limitations or other factors, measurements were not recorded on all patients (see Figure 1). The study was approved by the Ethics Committee of the Autonomous University of Nuevo Leon and the University of Michigan; all of the participants signed informed consent.

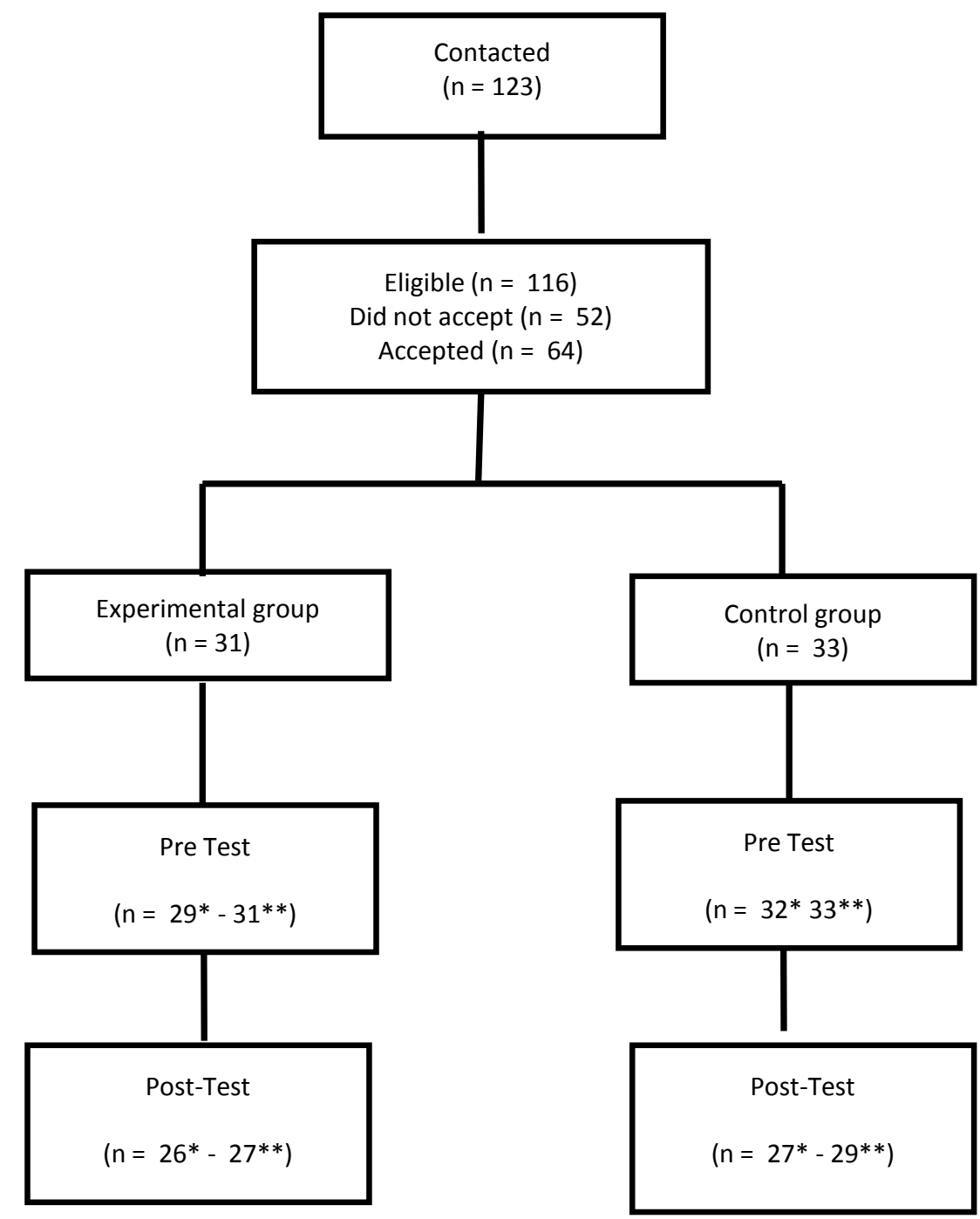

Number of participants with $\mathrm{HbAlc}$ measurements* and questionnaires ${ }^{* *}$

Figure 1. Diagram of the participants. 


\subsection{The Intervention}

The "Automated Telephone Diabetes Management" system [24] designed for Spanish-speaking groups residing in the USA (predominantly Mexican Americans) with similar characteristics [20] [25] to the selected sample was used in this study. The service was provided by "Quality Improvement for Complex Chronic Conditions" of the University of Michigan, Ann Arbor Medical School. The system intended to contact each patient in the intervention group weekly for 12 weeks. Patients received the telephone calls at an hour of their convenience, and the calls would last approximately 10 minutes. Patients used the digital buttons on their telephones to answer questions regarding to different aspects of their health state, need for bed rest, glucose monitoring, medication among others , using numbers solicited by the operator depending on the question (example, $1=$ yes, $2=$ no).

If the participant would allow, a health-promoting message would be played at the end (e.g., kind of food they may avoid and which should eat). Participants in the experimental group kept attending the medical appointments as programmed with their health care provider.

\subsection{Counseling}

Based on the information reported, the system would automatically generate two types of feedbacks for the medical team: information regarding the health of the person and information regarding the adherence to the self-care plan. This information was sent through email automatically to the administrator in charge of analyzing the data and channeling the requirements to either the physician or the nurse. When the patient reported problems monitoring glucose, hyper- or hypoglycemic symptoms, or was bed-bound due to illness, the clinical nurse would personally contact the patient and the caregiver via telephone. The nurse would also contact the patient personally when the system reported two unanswered automated telephone calls, or an alert was generated by the system based in the participants' report of low or high sugar, or other type of illnesses or discomfort.

\subsection{Training Participants}

Participants were trained to use the mobile telephone to receive the calls. This training consisted of indicating to the patient that the call would give them several reply options and that each option would be assigned a number that they would need to press according to the chosen reply. To ensure an understanding of the system and how to answer, the first telephone call of every participant was completed in the presence of the clinical nurse.

\subsection{Training the Research Team}

The training of the research team integrated by project manager and case nurse management was performed in the Department of Quality Improvement for 
Complex Chronic Conditions of the University of Michigan, Ann Arbor, Medical School. During the two and a half days of training, a review and the indications of the use of the questionnaires for the research were performed. The researchers were trained on the automated telephone system in the following areas: system components, general data entry of the participants in the system, programming telephone calls, reception of alerts and information from the participants.

\subsection{Key Measures and Variables}

Glycated hemoglobin ( $\mathrm{HbAlc}$ ) was measured in the subjects of the intervention and comparison groups at the beginning of the study and on week 12; processing of this biochemical marker was performed with chromatography method of high resolution (HPLC equipment D-10 Biorad). Blood pressure, weight and height (to calculate the body mass index, $[\mathrm{BMI}]$ ) and the waist circumference were measured at the beginning of the study following standard procedures. Patients from both groups completed three questionnaires when entering the study and on week 12. The Short Form Health Survey (SF12) scale [26], the Depression Symptoms Scale (CES-D) [27], and the Diabetes Self-Care scale [21]. Reliability of these instruments is reported in Table 1.

The group that received the automated calls responded to a final survey to evaluate their satisfaction with the service and the service's perceived impact on self-care. The satisfaction with the system was measured using four items as follows: (a) usefulness of the content of the telephone calls in T2D management; (b) improvement in the control of the disease due to the program; (c) accessibility and usefulness of the system; and (d) usefulness of the information with respect to self-management. The system automatically registered the date and time of each attempt and of each response. This information was used to calculate the call completion rates.

The acceptance of the system was evaluated in two focus group discussions; these sessions were held in two clinics in the middle of the program with 13 participants. The administrator of the intervention directed the sessions while the clinical nurses transcribed the notes. The questions were focused on the usefulness

Table 1. Instruments psychometric characteristics.

\begin{tabular}{lcc}
\multicolumn{1}{c}{ Instrument } & Items & Alpha Cronbach \\
\hline Short form health survey & 11 & 0.734 \\
Depressive symptoms scale & 9 & 0.777 \\
Diabetes self care (Importance of care) & 2 & 0.636 \\
Diabetes self care (Complications in treatment) & 4 & 0.700 \\
Diabetes self-care (Treatment relevance) & 7 & 0.657 \\
Diabetes self care (Ability) & 4 & 0.929 \\
Diabetes self care (Quiet medical treatment) & 4 & 0.619 \\
\hline
\end{tabular}


of and satisfaction with the program, the benefits of counting on a partner, and whether the patients experienced difficulties receiving the telephone calls; the discussions gave space for questions from the participants at the end of the sessions. Data was analyzed through content analysis, which gave the qualitative dimension to evaluate participants' satisfaction with the system.

\subsection{Analysis}

Central tendency and dispersion measures were calculated to represent the scores obtained from questionnaires. The effects on self-management, depression, and $\mathrm{HbAlc}$ levels were evaluated using t-Student statistics, comparing the baseline measure and the week 12 measure in the intervention and comparison groups. The results were considered significant when $p<0.05$. The data were processed using SPSS version 20 . The satisfaction scores and complications with the system were analyzed through descriptive statistics, also evaluated qualitatively (content analysis) using the data obtained from the focus group discussions.

\section{Results}

\subsection{Characteristics of the Participants}

Twenty-eight percent of the participants were male, $72 \%$ were female, and patient ages ranged from 28 to 82 years old $(M=56$ years; $\mathrm{SD}=13.27)$. The mean health literacy was $4.19(\mathrm{SD}=2.08$; interval $2-10)$; this is an important variable in T2D patients conducting self-care because it measures the understanding of the directions given by the health professionals.

Clinically, a high percentage of patients were overweight (BMI $>25$ and $<30$; $41.9 \%$ ) and exhibited general obesity (BMI $>30 ; 48.4 \%)$. Abdominal obesity was observed in $67 \%$ of the men and $89 \%$ of the women. Systolic and diastolic hypertension was observed in $23 \%$ and $37 \%$ of the participants, respectively. The intervention and comparison groups were equivalent at the beginning of the study in most demographic and clinical variables, as shown in Table 2.

Table 2. Demographic and clinical characteristics of the participants.

\begin{tabular}{|c|c|c|c|c|c|c|c|c|c|c|c|}
\hline \multirow{2}{*}{ Variables } & \multicolumn{5}{|c|}{ Intervention } & \multicolumn{5}{|c|}{ Comparison } & \multirow{2}{*}{$P$} \\
\hline & $n$ & $M$ & SD & Min & $\operatorname{Max}$ & $n$ & $M$ & SD & Min & $\operatorname{Max}$ & \\
\hline Age & 31 & 54.00 & 11.79 & 32 & 78 & 33 & 57.94 & 14.43 & 28 & 82 & 0.231 \\
\hline Schooling & 27 & 7.70 & 3.31 & 1 & 16 & 29 & 6.62 & 3.16 & 1 & 13 & 0.217 \\
\hline NPLH & 31 & 4.84 & 1.91 & 1 & 9 & 33 & 4.00 & 1.69 & 1 & 8 & 0.068 \\
\hline NFMLH & 31 & 2.68 & 1.27 & 1 & 6 & 33 & 2.00 & 1.03 & 01 & 4 & 0.026 \\
\hline Family income $e^{*}$ & 26 & 2202.19 & 1769.93 & 80 & 8700 & 25 & 1592.5 & 997.52 & 20.0 & 3500 & 0.138 \\
\hline $\mathrm{HbAlc}$ & 29 & 7.41 & 1.23 & 5.1 & 11.0 & 32 & 7.45 & 1.37 & 5.0 & 12.0 & 0.90 \\
\hline BMI & 31 & 29.77 & 3.87 & 22.02 & 38.33 & 33 & 29.59 & 7.17 & 18.24 & 49.99 & 0.90 \\
\hline
\end{tabular}

Notes: NPLH = Number of people living in the household; NFMLH = Number of family members living in the household; BMI = Body mass index; HbAlc $=$ Glycated hemoglobin (\%); ${ }^{\star}$ Weekly household income in Mexican Pesos. 


\subsection{Response of the Participants to the Use of the System}

Three hundred and seventy two telephone calls were programmed considering 12 weeks by 31 participants. Of these, 234 were completed (138 were missed or incomplete) resulting in a $62.9 \%$ response rate. However, the system performed 1,373 attempts to communicate, which means that there were 5.86 attempts per effective call. The reason for failed communication $(n=1139)$ included no response calls, somebody would answer but would hang up immediately, and technical failure.

\subsection{Satisfaction with the Intervention}

Focal group discussions provided information on aspects that would help with the treatment and with expressions that reflected satisfaction with the system and its impact on depression, as well as highlighted the barriers to the automated telephone system. With respect to the benefits in treatment, participants expressed that the calls "would help me remember to take the medication"; "I learned that I had to examine my feet"; "it reminds me to look after myself... that our body is important"; and "they remind me to check out glucose levels... and tell me the normal levels of glucose to know whether I'm doing well or not." With this system, satisfaction and the impact on depression was perceived through expressions such as the following: "I feel important to be part of the group"; "we can help each other learn about the disease and live longer"; "it helps us value ourselves"; "it helped me when I felt sad"; "my sister is my partner and now she is watching over me... before, nobody worried about me"; and "I am glad that my daughter calls me... now it feels as though she is more interested in knowing how I feel."

With respect to barriers, the following information was gathered: "I didn't know how to use the cellular phone"; "I had never had a cellular phone... I had problems answering the first calls"; "I would run out of battery in the middle of a phone call"; "my husband answered and hung up because he didn't know about the calls; he thought it was a salesman"; "I touched the wrong keys when answering"; and "I was not there; I forgot the time of the phone call."

The answers from the satisfaction survey demonstrated that $60 \%$ of the participants found the automated telephone calls to be helpful in controlling blood glucose, blood pressure, medication intake, and management of diet and health in general. Between $11 \%$ and $15 \%$ of the participants commented that they found the calls helpful "sometimes" and "usually," respectively.

\subsection{Effects of the Intervention on Physiological Control and Self-Care}

HbA1c measurements decreased $0.82 \%(M=7.41-6.69 ; t=25 ; 4.11 ; p=-0.001)$ from the baseline values over the 12 -week study in the intervention group and $0.49 \%(M=7.24-6.75 ; t=26 ; 2.11 ; p=0.044)$ in the comparison group. No significance difference $(t=51 ;-0.58 ; p=0.55)$ was observed when comparing the 
intervention and comparison groups with respect to the second measure. These data suggest a relationship of the automated calls on maintaining their diabetes self-care.

The baseline and 12-week measurements did not differ with respect to self-care health activities. The raw scores were low on scales involving the perception of the importance of health care, difficulties regarding the treatment, and medication intake. However, the capacity for self-care and the frequency of forgetting medications exhibited high values in the scale meaning the participants perceived themselves capable to manage their treatment (Table 3 ).

Variables related to medication intake did not exhibit differences between the baseline and second measurements, except with respect to the scale of depression; the intervention group exhibited a significant decrease compared with the comparison group $(t=53=-3.00, p=0.004 ; \mathrm{Ms}=20.58, \mathrm{SD}=5.36$ vs. $15.85, \mathrm{SD}$ $=2.07$ ).

Both groups perceived a low health status post-intervention, with no difference in the average scores $(\mathrm{Ms}=23.07, \mathrm{SD}=3.92$ and $24.96, \mathrm{SD}=7.20$, intervention and comparison groups, respectively).

\section{Discussion}

The main finding in this pilot study was that automated telephone calls are a feasible strategy to monitor treatment accomplishment, and performance of self-care in adults with $\mathrm{T} 2 \mathrm{D}$ and can promote the self-management of this chronic disease. The response rate to the programmed calls was similar to studies performed in the United States of America and in Latin American countries [20] [22] [25] [28]. Accordingly, participants' satisfaction suggests that this study could be performed on a large scale in Mexico with a good probability of obtaining positive results in the metabolic control of T2D patients.

The level of self-care activities did not vary significantly between the baseline and 12-week measures, which could suggest that the intervention was ineffective. A possible explanation is that the preparation received during the routine medical examinations (the consultation with the physician and educational sessions

Table 3. Average sub-scale self-care scores in T2D.

\begin{tabular}{|c|c|c|c|c|c|c|c|c|c|}
\hline \multirow[b]{2}{*}{ Sub-scale } & \multirow[b]{2}{*}{ Raw score } & \multicolumn{4}{|c|}{$\mathrm{T} 1$} & \multicolumn{4}{|c|}{$\mathrm{T} 2$} \\
\hline & & \multicolumn{2}{|c|}{ IG $n=31$} & \multicolumn{2}{|c|}{$\mathrm{CG} n=33$} & \multicolumn{2}{|c|}{ IG $n=27$} & \multicolumn{2}{|c|}{ CG $n=29$} \\
\hline Importance of care & $3-15$ & 9.38 & 2.91 & 9.42 & 3.06 & 8.00 & 2.11 & 9.00 & 2.50 \\
\hline Complications in treatment & $5-25$ & 16.74 & 5.42 & 17.27 & 5.28 & 18.14 & 4.99 & 21.34 & 19.54 \\
\hline Treatment relevance & $9-54$ & 36.77 & 6.67 & 34.00 & 7.53 & 30.77 & 6.64 & 30.62 & 8.02 \\
\hline Self-care ability & $4-24$ & 20.83 & 3.80 & 22.09 & 2.65 & 21.92 & 2.30 & 21.62 & 3.48 \\
\hline Quit medical treatment & $5-10$ & 8.03 & 1.12 & 7.93 & 1.45 & 8.23 & 1.14 & 8.25 & 1.55 \\
\hline
\end{tabular}

Note: Non-significant and inter-group mean differences; Intervention Group; CG = Comparison Group. 
with the nurses and nutrition professionals, as well as the occasional foot care team) is not sufficient to promote self-care and self-management of T2D patients. Patients express a low level of understanding of the instructions received, which could be a factor in this situation [29]. Several research studies present models that consider a protocolized educational event prior to the phone calls, which could help to confirm the level of understanding of essential skills of self-management [30]. Several authors support this idea and express the need to develop specialized skills for T2D treatment. Hyperglycemia usually produces a metabolic disorder unnoticed by the patient with T2D. This ignorance could lead to poorly administered hypoglycemic medication and to an inappropriate nutritional and exercise plan [31]. In conjunction with an automated telephone service such as the one studied here, this type of education may guarantee the understanding of the patients with respect to their condition to maximize the response and validity of the clinical data obtained from the patients during the phone calls.

The levels of $\mathrm{HbAlc}$ from both the intervention and comparison groups were better than the observed values in similar groups from the metropolitan area of Monterrey [29]. The sub-scale score of medication management of the participants in both groups was high, which may mean that treatment is followed at the time and indicated doses in the majority of cases. This could explain why the post-intervention measurement did not differ between the groups because both groups received their hypoglycemic medications on a strict schedule and at particular doses.

The level of depression improved significantly in the intervention group. These results were observed despite the fact that the symptoms and treatment of this condition were not mentioned in the phone calls. Interventions with automated phone calls in hypertensive patients [32] and in diabetic or hypertensive patients in the United States of America, Honduras and Mexico [20] [28] reported similar results. From the information gathered from the focus group, we believe that this effect is due to a feeling of "protection" from the members of the team including the case nurse management. Some researchers have reported that poor self-care in T2D treatment in adults may be secondary to a sustained depressive state with biochemical effects on the hypothalamus [33] [34]. This relationship suggests that the automated telephone management may be an important and positive intervention in these patients.

A significant result of this feasibility study was to assess the possibility of patient adherence to automated telephone protocols and the ability of primary care clinics where T2D ambulatory patients are treated to incorporate this model. For the participants, the main complications of this system were related to the poor state of the mobile phones, battery charge shortage or failure, and an inability to manipulate the device. Primary care clinics lack computer devices and telephone lines as well as a comprehensive care protocol for T2D patients with trained nurses that focuses on the development of self-care skills. These aspects should 
be considered in the design of the proposed model in the near future.

Possible limitations in this pilot study include the short duration of the intervention and the failure to include other important variables from diabetic patients, such as lipid count, sensitivity in lower extremities, and vision, as well presence of co-morbidities. It is important to emphasize the minimal training that the patients received in managing the reception of the automated calls.

\section{Conclusion}

The results of the current study suggest that it is feasible to use a healthcare model with automated phone calls as an educational and mid- and long-term follow-up strategy in patients with T2D. This model should be re-designed and include an educational curriculum, minimal computer equipment, internet and telephone lines in the primary care clinics, and formal training for the nurses in charge of the cases. The findings indicate that these services are successful for patients with low educational levels and that may assist in reducing depression symptoms.

\section{References}

[1] Villalpando, S., de la Cruz, V., Rojas, R., Shamah-Levy, T., Avila, M.A., Gaona, B., Rebollar, R. and Hernández, L. (2010) Prevalence and Distribution of Type 2 Diabetes Mellitus in Mexican Adult Population: A Probabilistic Survey. Salud Pública de México, 52, S19-S26. https://doi.org/10.1590/S0036-36342010000700005

[2] González-Villalpando, C., López-Ridaura, R., Campuzano, J. and González-Villalpando, M.E. (2010a) The Status of Diabetes Care in Mexican Population: Are We Making a Difference? Results of the National Health and Nutrition Survey 2006. Revista de Salud Pública de México, 52, S36-S43. https://doi.org/10.1590/S0036-36342010000700007

[3] González-Villalpando, C., López-Ridaura, R., Lazcano-Ponce, E. and González-Villalpando, M.E. (2010b) And Now What? Time for Daring Innovation. Revista de Salud Pública de México, 52, S80-S83.

[4] Vadstrup, E., Frolich, A., Perrild, H., Borg, E. and Roder, M. (2009) Lifestyle Intervention for Type 2 Diabetes Patients-Trial Protocol of the Copenhagen Type 2 Diabetes Rehabilitation Project. BMC Public Health, 9, 166-173. https://doi.org/10.1186/1471-2458-9-166

[5] Tuomilehto, J., Schwarz, P. and Lindstrom, J. (2011) Long-Term Benefits from Lifestyle Interventions for Type 2 Diabetes Prevention. Diabetes Care, 34, S210-S214. https://doi.org/10.2337/dc11-s222

[6] Vermeire, E., Wens, J., Van Royen, P., Biot, Y., Hearnshaw, H. and Lindenmeyer, A. (2005) Interventions for Improving Adherence to Treatment Recommendations in People with Type 2 Diabetes Mellitus. The Cochrane Database of Systematic Reviews, 18, CD003638.

[7] Keogh, K.M., White, P., Hevey, D., McGilloway, S. and Smith, S.M. (2007) Family Based Interventions to Improve Outcomes in Patients with Type 2 Diabetes Mellitus. Cochrane Database of Systematic Reviews, 1, CD006382.

[8] Wu, L., Forbes, A., Griffiths, P. and While, A. (2009) Telephone Follow-Up for Type 2 Diabetes Mellitus. Cochrane Database of Systematic Reviews, 3, CD006694.

[9] Faridi, Z., Liberti, L., Shuval, K., Northrup, V., Ali, A. and Katz, D.L. (2008) Eva- 
luating the Impact of Mobile Telephone Technology on Type 2 Diabetic Patients' Self-Management: The NICHE Pilot Study. Journal of Evaluation in Clinical Practice, 14, 465-469.

[10] Handley, M.A., Shumway, M. and Schillinger, D. (2008) Cost-Effectiveness of Automated Telephone Self-Management Support with Nurse Care Management among Patients with Diabetes. Annals of Family Medicine, 6, 512-518. https://doi.org/10.1370/afm.889

[11] Bird, D., Oldenburg, B., Cassimatis, M., Russell, A., Ash, S., Courtney, M.D., Scuffham, P.A., Stewart, I., Wootton, R. and Friedman, R.H. (2010) Randomized Controlled Trial of an Automated, Interactive Telephone Intervention to Improve Type 2 Diabetes Self-Management (Telephone-Linked Care Diabetes Project): Study Protocol. BMC Public Health, 10, 599. https://doi.org/10.1186/1471-2458-10-599

[12] Liang, X., Wang, Q., Yang, X., Cao, J., Chen, J., Mo, X., Huang, J., Wang, L. and Gu, D. (2010) Effect of Mobile Phone Intervention for Diabetes on Glycaemic Control: A Meta-Analysis. Diabetic Medicine, 28, 455-463. https://doi.org/10.1111/j.1464-5491.2010.03180.x

[13] Robbins, J.M., Vaccarino, V., Zhang, H. and Kasl, S.V. (2001) Socioeconomic Status and Type 2 Diabetes in African American and Non-Hispanic White Women and Men: Evidence from the Third National Health and Nutrition Examination Survey. American Journal of Public Health, 91, 76-83. https://doi.org/10.2105/AJPH.91.1.76

[14] Rosal, M., Ockene, I., Restrepo, A., White, M., Borg, A., Olendzki, B., Scarvron, J., Candib, L., Welch, G. and Reed, G. (2011) Randomized Trial of a Literary-Sensitive, Culturally Tailored Diabetes Self-Management Intervention for Low Income Latino. Diabetes Care, 34, 838-844. https://doi.org/10.2337/dc10-1981

[15] Federal Commission of Telecommunications (2012) Estadísticas de Comunicación, [Communication Statistics.] Federal Commission of Telecommunications.

[16] Kim, H.S. and Jeong, H.S. (2007) A Nurse Short Message Service by Cellular Phone in Type-2 Diabetic Patients for Six Months. Journal of Clinical Nursing, 16, 1082-1087. https://doi.org/10.1111/j.1365-2702.2007.01698.x

[17] Yoon, K.H. and Kim, H.S. (2008) A Short Message Service by Cellular Phone in Type 2 Diabetic Patients for 12 Months. Diabetes Research and Clinical Practice, 79, 256-261. https://doi.org/10.1016/j.diabres.2007.09.007

[18] Vervloet, M., van Dijk, L., Santen-Reestman, J., van Vlijmen, B., Bouvy, M.L. and de Bakker, D.H. (2011) Improving Medication Adherence in Diabetes Type 2 Patients through Real Time Medication Monitoring: A Randomized Controlled Trial to Evaluate the Effect of Monitoring Patients Medication Use Combined with Short Message Service (SMS) Reminders. BMC Health Services Research, 11, 5. https://doi.org/10.1186/1472-6963-11-5

[19] Piette, J.D., Moura, L.A., Fraser, H.S., Mechael, P.N., Powell, J. and Khoja, S.R. (2012b) Impacts of E-Health on the Outcomes of Care in Low- and Middle-Income Countries: Where Do We Go from Here? WHO Bulletin, 90, 365-372. https://doi.org/10.2471/BLT.11.099069

[20] Piette, J.D., Weinberger, M. and McPhee, S.J. (2000) The Effect of Automated Calls with Telephone Nurse Follow-Up on Patient-Centered Outcomes of Diabetes Care: A Randomized, Controlled Trial. Medical Care, 38, 218-230. https://doi.org/10.1097/00005650-200002000-00011

[21] Loring, K., Ritter, P., Villa, F. and Piette, J. (2008) Spanish Diabetes Self-Management with and without Automated Telephone Reinforcement. Diabetes Care, 31, 408-414. https://doi.org/10.2337/dc07-1313 
[22] Piette, J.D., Mendoza-Avelares, M.O., Ganser, M., Mohamed, M., Marinec, N. and Krishnan, S. (2011) A Preliminary Study of a Cloud-Computing Model for Chronic Illness Self-Care Support in an Underdeveloped Country. American Journal of Preventive Medicine, 40, 629-632. https://doi.org/10.1016/j.amepre.2011.02.014

[23] Piette, J.D., Marinec, N., Gallegos-Cabriales, E.C., Gutierrez-Valverde, J.M., Rodriguez-Saldaña, J., Mendoz-Alevares, M. and Silveira, M.J. (2013) Spanish-Speaking Patients' Engagement in Interactive Voice Response (IVR) Support Calls for Chronic Disease Self-Management: Data from Three Countries. Journal of Telemedicine and Telecare, 19, 89-94. https://doi.org/10.1177/1357633x13476234

[24] Piette, J.D. (2000) Satisfaction with Automated Telephone Disease Management Calls and Its Relationship to Their Use. The Diabetes Educator, 26, 1003-1010. https://doi.org/10.1177/014572170002600613

[25] Piette, J.D., McPhee, S.J., Weinberger, M., Mah, C.A. and Kraemer, F.B. (1999) Use of Automated Telephone Disease Management Calls in an Ethnically Diverse Sample of Low-Income Patients with Diabetes. Diabetes Care, 22, 1302-1309. https://doi.org/10.2337/diacare.22.8.1302

[26] Cisneros-Huerta, A. (2007) Validez y confiabilidad del cuestionario general de salud (SF 12) en derechohabientes de unidad de primer nivel del IMSS. Universidad Veracruzana. [Validity and Reliability of the General Questionnaire on Health [SF 12] with Beneficiary Patients of the Mexican Social Security an Ambulatory Clinic.]

[27] Radloff, L.S. (1997) The CES-D Scale: A Self-Report Depression Scale for Research in the General Population. Applied Psychological Measurement, 1, 385-340. https://doi.org/10.1177/014662167700100306

[28] Piette, J.D., Datwani, H., Gaudioso, S., Foster, S.M., Westphal, J., Perry, W., Rodríguez-Saldaña, J., Mendoza-Avelares, M.O. and Marinec, N. (2012a) Hypertension Management Using Mobile Technology and Home Blood Pressure Monitoring: Results of a Randomized Trial in Two Low/Middle Income Countries. Telemedicine Journal and E-Health, 18, 613-620. https://doi.org/10.1089/tmj.2011.0271

[29] Gallegos, E.C., Berúmen, F. and Gómez-Meza, M.V. (2006) Metabolic Control of Adults with Type 2 Diabetes Mellitus through Education and Counseling. Journal of Nursing Scholarship, 38, 344-351. https://doi.org/10.1111/j.1547-5069.2006.00125.x

[30] Pal, K., Eastwood, S.V., Michie, S., Farmer, A.J., Barnard, M.L., Peacock, R., Wood, B., Inniss, J.D. and Murray, E. (2010) Computer-Based Diabetes Self-Management Interventions for Adults with Type 2 Diabetes Mellitus. Cochrane Database of Systematic Reviews, 3, CD008776. https://doi.org/10.1002/14651858.CD008776

[31] Vermeire, E., Hearnshaw, H., Rätsep, A., Levasseur, G., Petek, D., van Dam, H., van der Horst, F., Vinter-Repalust, N., Wens, J., Dale, J. and Van Royen, P. (2007) Obstacles to Adherence in Living with Type-2 Diabetes: An International Qualitative Study Using Meta-Ethnography (EUROBSTACLE). Primary Care Diabetes, 1, 25-33. https://doi.org/10.1016/j.pcd.2006.07.002

[32] Krishna, S. and Austin, S. (2008) Diabetes Self-Management Care via Cell Phone: A Systematic Review. Journal of Diabetes Science Technology, 2, 139-146. https://doi.org/10.1177/193229680800200324

[33] Wagner, J.A., Tennen, H. and Osbornt, Y. (2010) Lifetime Depression and Diabetes Self-Management in Women with Type 2 Diabetes: A Case-Control Study. Diabetic Medicine, 27, 713-717. https://doi.org/10.1177/193229680800200324

[34] Gragnoli, C. (2011) Depression and Type 2 Diabetes: Cortisol Pathway Implication and Investigational Needs. Journal of Cellular Physiology, 227, 2318-2322. https://doi.org/10.1002/jcp.23012 